\title{
Ground States for the Schrödinger Systems with Harmonic Potential and Combined Power-Type Nonlinearities
}

\author{
Baiyu Liu \\ School of Mathematics and Physics, University of Science and Technology Beijing, 30 Xueyuan Road, \\ Haidian District, Beijing 100083, China
}

Correspondence should be addressed to Baiyu Liu; liubymath@gmail.com

Received 14 June 2014; Accepted 28 August 2014; Published 19 October 2014

Academic Editor: Vladimir Georgiev

Copyright (C) 2014 Baiyu Liu. This is an open access article distributed under the Creative Commons Attribution License, which permits unrestricted use, distribution, and reproduction in any medium, provided the original work is properly cited.

We consider a class of coupled nonlinear Schrödinger systems with potential terms and combined power-type nonlinearities. We establish the existence of ground states, by using a variational method. As an application, some symmetry results for ground states of Schrödinger systems with harmonic potential terms are obtained.

\section{Introduction}

In this paper, we are interested in the steady state of the coupled nonlinear Schrödinger system

$$
\begin{aligned}
-i \varphi_{1 t}= & \Delta \varphi_{1}-Q_{1}(x) \varphi_{1}+\mu_{1}\left|\varphi_{1}\right|^{p} \varphi_{1} \\
& +\sum_{k=1}^{m} \alpha_{k}\left|\varphi_{1}\right|^{p_{k}-2}\left|\varphi_{2}\right|^{q_{k}} \varphi_{1}, \\
-i \varphi_{2 t}= & \Delta \varphi_{2}-Q_{2}(x) \varphi_{2}+\mu_{2}\left|\varphi_{2}\right|^{p} \varphi_{2} \\
& +\sum_{k=1}^{m} \beta_{k}\left|\varphi_{1}\right|^{p_{k}}\left|\varphi_{2}\right|^{q_{k}-2} \varphi_{1},
\end{aligned}
$$

in $\mathbb{R}^{N}$, where $\mu_{k}, \alpha_{k}, \beta_{k}, p_{k}$, and $q_{k}$ are real constants, $p_{k}+q_{k}=$ $p+2, \alpha_{k} q_{k}=\beta_{k} p_{k}(k=1,2, \ldots, m)$, and $1<p<4 /(N-2)^{+}$, $\left((N-2)^{+}=N-2\right.$ when $N \geq 3$ and $4 /(N-2)^{+}=\infty$ when $N=$ $1,2)$. System (1) has applications in many physical problems, especially in the Hartree-Fock theory for a double BoseEinstein condensate with interparticle interactions under the magnetic trap. In physic, $Q_{i}(x)$ is the trapping potential for the $i$ th species, whose role is to confine the movement of particles. We remark that the harmonic potential $|x|^{2}$ is a widely used trapping potential in current experiments [1].
We call solutions of forms $\varphi_{1}(x, t)=e^{i \omega_{1} t} u(x), \varphi_{2}(x, t)=$ $e^{i \omega_{2} t} v(x)\left(\omega_{i}>0\right)$ standing wave solutions to $(1)$, where $(u, v)$ solves the following elliptic system:

$$
\begin{aligned}
& -\Delta u+V_{1}(x) u=\mu_{1}|u|^{p} u+\sum_{k=1}^{m} \alpha_{k}|u|^{p_{k}-2}|v|^{q_{k}} u, \\
& -\Delta v+V_{2}(x) v=\mu_{2}|v|^{p} v+\sum_{k=1}^{m} \beta_{k}|u|^{p_{k}}|v|^{q_{k}-2} v .
\end{aligned}
$$

Here $V_{i}(x)=Q_{i}(x)+\omega_{i}(i=1,2)$.

The energy functional of (2) is

$$
\begin{array}{r}
I(u, v):=\frac{1}{2} \int_{\mathbb{R}^{N}}\left(|\nabla u|^{2}+V_{1}(x) u^{2}+|\nabla v|^{2}+V_{2}(x) v^{2}\right) \\
-\frac{1}{p+2} \int_{\mathbb{R}^{N}}\left(\mu_{1}|u|^{p+2}+\mu_{2}|v|^{p+2}\right. \\
\left.+\sum_{k=1}^{m}\left(\alpha_{k}+\beta_{k}\right)|u|^{p_{k}}|v|^{q_{k}}\right)
\end{array}
$$

and the work space $E:=\left\{(u, v) \in H^{1}\left(\mathbb{R}^{N}\right) \times H^{1}\left(\mathbb{R}^{N}\right) \mid\right.$ $\left.\int_{\mathbb{R}^{N}} V_{1}(x) u^{2} d x+\int_{\mathbb{R}^{N}} V_{2}(x) v^{2} d x<\infty\right\}$. Since $E \hookrightarrow$ $L^{p+2}\left(\mathbb{R}^{N}\right) \times L^{p+2}\left(\mathbb{R}^{N}\right)$ compactly (for $0 \leq p<4 /(N-2)$ ) and $E$ is a Hilbert space with norm $\|(u, v)\|_{E}^{2}=\int_{\mathbb{R}^{N}}\left(|\nabla u|^{2}+\right.$ 
$\left.V_{1}(x) u^{2}\right) d x+\int_{\mathbb{R}^{N}}\left(|\nabla v|^{2}+V_{2}(x) v^{2}\right) d x$ (see $\left.[2,3]\right)$, the energy functional $I(u, v)$ makes sense in the work space.

We say that $(u, v) \in E$ is a nontrivial $(u \neq \equiv 0$ or $v \neq \equiv 0)$ bound state of $(2)$ if $(u, v)$ is a nontrivial critical point of $I$. The ground state is usually defined as the positive minimizer of the following minimization problem:

$$
\min _{(w, z) \in E}\{I(w, z) \mid d I(w, z)=0\} .
$$

The existence and structure of ground states for Schrödinger equation or systems have been investigated by many authors (see [4-19] and the references therein).

In the case of a single nonlinear Schrödinger equation $-\Delta u=f(u), x \in \mathbb{R}^{N}$, under some appropriate conditions on $f$, the ground state exists and is radially symmetric $[5,8,19]$. For the single equation with potential terms

$$
-\Delta u+V(x) u=f(x, u), \quad u>0, \text { in } \mathbb{R}^{N},
$$

Rabinowitz [14] used variational methods based on variants of mountain pass theorem to prove that (5) has a positive ground state, for those $V(x)$ satisfying $\lim _{|x| \rightarrow \infty} V(x)=+\infty$ and $V(x) \geq c>0$. Similar results were also obtained in [6].

In the case of nonlinear Schrödinger systems, Lin and Wei [11] considered two-component systems of nonlinear Schrödinger equations with trap potentials:

$$
\begin{gathered}
-\epsilon^{2} \Delta u_{j}+V_{j}(x) u_{j}=\sum_{j=1}^{2} \beta_{i j} u_{i}^{2} u_{j}, \quad x \in \mathbb{R}^{N}, \\
u_{j}>0, \quad u_{j} \in H^{1}\left(\mathbb{R}^{N}\right), \quad j=1,2,
\end{gathered}
$$

where $N=2,3, \epsilon>0$. Among other things, they showed that there is some $\beta_{0}>0$ such that if $\beta_{11}>0, \beta_{22}>0, \beta_{12}=\beta_{21} \in$ $\left(-\infty, \beta_{0}\right), \lim _{|x| \rightarrow \infty} V_{j}(x)=+\infty$, and inf ${ }_{x \in \mathbb{R}^{N}} V_{j}>0, j=1,2$, then the ground state solution to (6) always exists.

Sirakov [17] studied a system of two equations,

$$
\begin{aligned}
& -\Delta u+u=\mu_{1} u^{3}+\beta u v^{2}, \quad x \in \mathbb{R}^{N}, \\
& -\Delta v+\lambda v=\mu_{2} v^{3}+\beta u^{2} v, \quad x \in \mathbb{R}^{N},
\end{aligned}
$$

and found that there are always ranges of positive parameters in (7), for which it has a ground state, and ranges of positive parameters for which it does not have ground state.

Maia et al. [13] considered the weakly couple nonlinear elliptic system

$$
\begin{aligned}
& -\Delta u+u=|u|^{2 q-2} u+b|v|^{q}|u|^{q-2} u, \quad x \in \mathbb{R}^{N}, \\
& -\Delta v+\omega^{2} v=|v|^{2 q-2} v+b|u|^{q}|v|^{q-2} v, \quad x \in \mathbb{R}^{N},
\end{aligned}
$$

where $1<q<N /(N-2)$ for $N \geq 3$ and $q>1$ for $N=1,2$. They showed that if $b$ is sufficiently large, then there exists a nontrivial positive ground state of (8).

$\mathrm{Ma}$ and Zhao [12] considered

$$
\begin{array}{ll}
-\Delta u+u=\mu_{1}|u|^{2 p} u+\beta_{1}|v|^{p+1}|u|^{p-1} u, & x \in \mathbb{R}^{N}, \\
-\Delta v+v=\mu_{2}|v|^{2 p} v+\beta_{2}|u|^{p+1}|v|^{p-1} v, & x \in \mathbb{R}^{N},
\end{array}
$$

in which $0<p<2 /(n-2)^{+}$. Under assumptions $\mu_{1}, \mu_{2} \leq 0$, $\beta_{1}, \beta_{2}>0, \mu_{1} \beta_{2}^{p}=\mu_{2} \beta_{2}^{p}$, and $\mu_{1}+\beta_{2}^{(p+1) / 2} / \beta_{1}^{(p-1) / 2}>0$, or $\mu_{2}+\beta_{1}^{(p+1) / 2} / \beta_{2}^{(p-1) / 2}>0$, then the ground state of (9) exists and is unique up to translations.

Song [18] obtained the existence of ground states for a system of Schrödinger equations with combined power-type nonlinearities and with no trap potentials. It is natural to consider similar results for Schrödinger system with potential terms and the combined power-type nonlinearities.

Motived by the above work, in this paper we focus on the existence and symmetric properties for the ground states of Schrödinger system (2). For simplicity, we only prove the existence result for ground state of

$$
\begin{aligned}
& -\Delta u+V(x) u=\mu|u|^{p} u+\sum_{k=1}^{m} \alpha_{k}|u|^{p_{k}-2}|v|^{q_{k}} u, \quad \text { in } \mathbb{R}^{N}, \\
& -\Delta v+V(x) v=\mu|v|^{p} v+\sum_{k=1}^{m} \beta_{k}|u|^{p_{k}}|v|^{q_{k}-2} v, \quad \text { in } \mathbb{R}^{N},
\end{aligned}
$$

where $1<p<4 /(N-2)^{+}, N \leq 5$, and $\mu_{k}, \alpha_{k}, \beta_{k}, p_{k}$, and $q_{k}$ are real constants satisfying

$$
\begin{gathered}
1<p_{k}<p+1, \quad 1<q_{k}<p+1, \\
p_{k}+q_{k}=p+2, \quad \alpha_{k} q_{k}=\beta_{k} p_{k}, \quad(k=1,2, \ldots, m) .
\end{gathered}
$$

To be precise, our first result reads as follows.

Theorem 1. Consider (11) and (12), where

$$
\begin{gathered}
\mu<\frac{\sum_{k=1}^{m}\left(\alpha_{k}+\beta_{k}\right)}{2\left(2^{p / 2}-1\right)}, \\
\mu>-\sum_{k=1}^{m} \min \left\{0, \alpha_{k}\right\}, \quad \mu>-\sum_{k=1}^{m} \min \left\{0, \beta_{k}\right\} .
\end{gathered}
$$

Suppose the potential term $V(x)$ satisfies

$$
\begin{gathered}
V(x) \in C^{1}\left(\mathbb{R}^{N}, \mathbb{R}\right), \quad V(x) \geq c_{0}>0, \\
\lim _{|x| \rightarrow \infty} V(x)=+\infty .
\end{gathered}
$$

Then the ground state of system (10) exists.

Remark 2. Condition (13) is the same as (1.10) and (1.11) in [18], in which Schrödinger systems (2) with $V(x) \equiv 0$ were considered. The result in [18] is suitable for the case with all coefficients being positive, while our result can be applied to system (2) with some negative $\alpha_{k}$ and $\beta_{k}$. 
As an application, we give a symmetric result for system with harmonic potential in $\mathbb{R}^{3}$ :

$$
\begin{array}{r}
-\Delta u+\left(|x|^{2}+1\right) u=\mu|u|^{p} u+\sum_{k=1}^{m} \alpha_{k}|u|^{p_{k}-2}|v|^{q_{k}} u, \\
\text { in } \mathbb{R}^{3}, \\
-\Delta v+\left(|x|^{2}+1\right) v=\mu|v|^{p} v+\sum_{k=1}^{m} \beta_{k}|u|^{p_{k}}|v|^{q_{k}-2} v, \\
\text { in } \mathbb{R}^{3},
\end{array}
$$

where $0<p<4$ and $\mu_{k}, \alpha_{k}, \beta_{k}, p_{k}$, and $q_{k}$ are real constants which satisfy (11), (12), (13), and (14). In this case the work space

$$
\begin{aligned}
\Sigma:=\{ & (u, v) \in H^{1}\left(\mathbb{R}^{3}\right) \\
& \left.\times H^{1}\left(\mathbb{R}^{3}\right) \mid \int_{\mathbb{R}^{3}}\left(|x|^{2}|u|^{2}+|x|^{2}|v|^{2}\right) d x<+\infty\right\} .
\end{aligned}
$$

We first show that each solution of (16) is classic and decays at infinity.

Theorem 3. Suppose $(u, v) \in \Sigma$ solves (16) with $2<p<4, \mu_{k}$, $\alpha_{k}, \beta_{k}, p_{k}$, and $q_{k}$ being real constants satisfying $2<p_{k}<p+1$, $2<q_{k}<p+1$, and $p_{k}+q_{k}=p+2$. Then
(a) $u, v \in W^{2, l}\left(\mathbb{R}^{3}\right)$, for every $2 \leq l<\infty$;
(b) $\lim _{|x| \rightarrow \infty} u(x)=0$ and $\lim _{|x| \rightarrow \infty} v(x)=0$;
(c) $u, v \in C^{2}\left(\mathbb{R}^{3}\right)$.

Using the above regularity and decay result, one can easily show that the ground state is radial symmetric.

Corollary 4. Assume $\alpha_{k}>0, \beta_{k}>0,2<p_{k}<p+1,2<q_{k}<$ $p+1, p_{k}+q_{k}=p+2$, (12), (13), and (14). Then there exists a radial symmetric ground state of system (16).

An outline of this paper is as follows. We devote Section 2 to some preparations and the proof of Theorem 1 . The proofs of Theorem 3 and Corollary 4 will be given in Section 3 .

\section{Existence of Ground State}

In this section, we always assume (11), (12), (13), (14), and (15). Also, we denote by $\|\cdot\|_{p}$ the $L^{p}\left(\mathbb{R}^{N}\right)$ norm for $1 \leq p \leq \infty$. Recall that the work space $E:=$ $\left\{(u, v) \in H^{1}\left(\mathbb{R}^{N}\right) \times H^{1}\left(\mathbb{R}^{N}\right) \quad \mid \int_{\mathbb{R}^{N}} V(x)\left(u^{2}+v^{2}\right) d x<\right.$ $\infty\}$ is a Hilbert space with norm $\|(u, v)\|_{E}=$ $\left(\int_{\mathbb{R}^{N}}\left(|\nabla u|^{2}+V(x) u^{2}\right) d x+\int_{\mathbb{R}^{N}}\left(|\nabla v|^{2}+V(x) v^{2}\right) d x\right)^{1 / 2}$.

We now introduce somelemmas, which will be needed in the proof of Theorem 1.

Lemma 5. All critical points of energy function $I(u, v)$ in $E$ are weak solutions of system (10).
Proof. Suppose $(u, v) \in E$ is a critical point of $I$; that is,

$$
d I(u, v)\left[\varphi_{1}, \varphi_{2}\right]=0, \quad \forall \varphi_{1}, \varphi_{2} \in E .
$$

Direct computation shows that, for all $\varphi_{1}, \varphi_{2} \in E$, we have

$$
\begin{aligned}
0= & d I(u, v)\left[\varphi_{1}, \varphi_{2}\right] \\
= & \lim _{\epsilon \rightarrow 0} \frac{1}{\epsilon}\left(I\left(u+\epsilon \varphi_{1}, v+\epsilon \varphi_{2}\right)-I(u, v)\right) \\
= & \int_{\mathbb{R}^{N}}\left(\nabla u \cdot \nabla \varphi_{1}+\nabla v \cdot \nabla \varphi_{2}\right. \\
& \left.\quad+V(x) u \cdot \varphi_{1}+V(x) v \cdot \varphi_{2}\right) d x \\
& -\int_{\mathbb{R}^{N}}\left(\mu|u|^{p} u \varphi_{1}+\mu|v|^{p} v \varphi_{2}\right) d x \\
& -\int_{\mathbb{R}^{N}} \frac{\sum_{k}\left(\alpha_{k}+\beta_{k}\right) p_{k}}{p+2}|u|^{p_{k}-2} u|v|^{q_{k}} \varphi_{1} d x \\
& -\int_{\mathbb{R}^{N}} \frac{\sum_{k}\left(\alpha_{k}+\beta_{k}\right) q_{k}}{p+2}|u|^{p_{k}}|v|^{q_{k}-2} v \varphi_{2} d x .
\end{aligned}
$$

Using (12), we have

$$
\frac{\sum_{k}\left(\alpha_{k}+\beta_{k}\right) p_{k}}{p+2}=\sum_{k} \alpha_{k}, \quad \frac{\sum_{k}\left(\alpha_{k}+\beta_{k}\right) q_{k}}{p+2}=\sum_{k} \beta_{k} .
$$

Hence, for all $\varphi_{1}, \varphi_{2} \in E$

$$
\begin{aligned}
0= & d I(u, v)\left[\varphi_{1}, \varphi_{2}\right] \\
=\int_{\mathbb{R}^{N}} & \left(\nabla u \cdot \nabla \varphi_{1}+V(x) u \cdot \varphi_{1}-\mu|u|^{p} u \cdot \varphi_{1}\right. \\
& \left.\quad-\sum_{k} \alpha_{k}|u|^{p_{k}-2} u|v|^{q_{k}} \cdot \varphi_{1}\right) d x \\
& +\int_{\mathbb{R}^{N}}\left(\nabla v \cdot \nabla \varphi_{2}+V(x) v \cdot \varphi_{2}-\mu|v|^{p} v \cdot \varphi_{2}\right. \\
& \left.\quad-\sum_{k} \beta_{k}|u|^{p_{k}}|v|^{q_{k}-2} v \cdot \varphi_{2}\right) d x .
\end{aligned}
$$

Therefore, $(u, v)$ is a weak solution of system $(10)$.

Define $T_{1}:=\{(u, v) \in E \mid u \neq \equiv 0$ or $v \not \equiv 0\}$ and the Nehari manifold

$$
\begin{aligned}
N_{1}:=\{(u, v) & \\
\in & T_{1} \mid \int_{\mathbb{R}^{N}}\left(|\nabla u|^{2}+V(x) u^{2}\right. \\
& \left.+|\nabla v|^{2}+V(x) v^{2}\right) d x \\
= & \int_{\mathbb{R}^{N}}\left(\mu|u|^{p+2}+\mu|v|^{p+2}\right. \\
& \left.\left.\quad+\sum_{k=1}^{m}\left(\alpha_{k}+\beta_{k}\right)|u|^{p_{k}}|v|^{q_{k}}\right) d x\right\} .
\end{aligned}
$$


Note that $N_{1} \neq \emptyset$ when (11), (12), (14), and (15) are satisfied. Actually, by using (12) and (14), one can prove that, for all $(u, v) \in T_{1}$,

$$
\int_{\mathbb{R}^{N}}\left(\mu|u|^{p+2}+\mu|v|^{p+2}+\sum_{k=1}^{m}\left(\alpha_{k}+\beta_{k}\right)|u|^{p_{k}}|v|^{q_{k}}\right) d x>0 .
$$

Therefore, we can choose

$$
\begin{aligned}
& t=(\left(\int_{\mathbb{R}^{N}}\left(|\nabla u|^{2}+V(x) u^{2}+|\nabla v|^{2}+V(x) v^{2}\right) d x\right) \\
& \times\left(\int _ { \mathbb { R } ^ { N } } \left(\mu|u|^{p+2}+\mu|v|^{p+2}\right.\right. \\
&\left.\left.\left.+\sum_{k=1}^{m}\left(\alpha_{k}+\beta_{k}\right)|u|^{p_{k}}|v|^{q_{k}}\right) d x\right)^{-1}\right)^{1 / p}>0
\end{aligned}
$$

such that $(t u, t v) \in N_{1}$.

The following lemma shows that distance between the Nehari manifold $N_{1}$ and $(0,0)$ is positive in $H^{1}\left(\mathbb{R}^{N}\right) \times H^{1}\left(\mathbb{R}^{N}\right)$ or in $L^{p+2}\left(\mathbb{R}^{N}\right) \times L^{p+2}\left(\mathbb{R}^{N}\right)$.

Lemma 6. Assume $1<p<4 /(N-2)^{+}, N \leq 5, \mu>0$, and (15). Then there are positive constants $\epsilon_{1}, \epsilon_{2}>0$, such that

$$
\begin{gathered}
\|u\|_{H^{1}\left(\mathbb{R}^{N}\right)}+\|v\|_{H^{1}\left(\mathbb{R}^{N}\right)} \geq \epsilon_{1}>0, \quad \forall(u, v) \in N_{1}, \\
\|u\|_{p+2}^{p+2}+\|v\|_{p^{+2}}^{p+2} \geq \epsilon_{2}>0, \quad \forall(u, v) \in N_{1} .
\end{gathered}
$$

Proof. Choose $0<e<\min \left\{c_{0}, 1\right\}$. For each $(u, v) \in N_{1}$, by using (15) and Young's inequality, we have

$$
\begin{aligned}
& e\left(\|u\|_{H^{1}\left(\mathbb{R}^{N}\right)}^{2}+\|v\|_{H^{1}\left(\mathbb{R}^{N}\right)}^{2}\right) \\
& \leq \int_{\mathbb{R}^{N}}\left(|\nabla u|^{2}+V(x) u^{2}+|\nabla v|^{2}+V(x) v^{2}\right) \\
& =\int_{\mathbb{R}^{N}}\left(\mu|u|^{p+2}+\mu|v|^{p+2}+\sum_{k=1}^{m}\left(\alpha_{k}+\beta_{k}\right)|u|^{p_{k}}|v|^{q_{k}}\right) d x \\
& \leq \mu\left(\|u\|_{p+2}^{p+2}+\|v\|_{p^{+2}}^{p+2}\right) \\
& \quad+\sum_{k=1}^{m}\left|\alpha_{k}+\beta_{k}\right| \int_{\mathbb{R}^{N}}\left(\frac{p_{k}}{p+2}|u|^{p+2}+\frac{q_{k}}{p+2}|v|^{p+2}\right) d x \\
& \leq C\left(\|u\|_{p+2}^{p+2}+\|v\|_{p+2}^{p+2}\right) .
\end{aligned}
$$

Since $H^{1}\left(\mathbb{R}^{N}\right)$ is embedded into $L^{p+2}\left(\mathbb{R}^{N}\right)(0<p<$ $\left.4 /(N-2)^{+}\right)$, we know that

$$
\begin{aligned}
\|u\|_{p+2}^{p+2}+\|v\|_{p+2}^{p+2} & \leq C^{\prime}\left(\|u\|_{H^{1}\left(\mathbb{R}^{N}\right)}^{p+2}+\|v\|_{H^{1}\left(\mathbb{R}^{N}\right)}^{p+2}\right) \\
& \left.\leq C^{\prime}\left(\|u\|_{H^{1}\left(\mathbb{R}^{N}\right)}^{2}+\|v\|_{H^{1}\left(\mathbb{R}^{N}\right)}^{2}\right)\right)^{p},
\end{aligned}
$$

where we have used the fact that $p>1$.
Therefore, combining the above inequality with (26), we obtain

$$
\|u\|_{H^{1}\left(\mathbb{R}^{N}\right)}^{2}+\|v\|_{H^{1}\left(\mathbb{R}^{N}\right)}^{2} \geq \epsilon_{1}:=\left(\frac{e}{C C^{\prime}}\right)^{1 /(p-1)}>0 .
$$

Putting (28) into (26), we obtain

$$
\|u\|_{p+2}^{p+2}+\|v\|_{p+2}^{p+2} \geq \epsilon_{2}:=\left(\frac{e}{C}\right) \epsilon_{1}>0 .
$$

For each $(u, v) \in T_{1}$, define

$$
f_{(u, v)}(t):=I(t u, t v), \quad t \in[0,+\infty) .
$$

Since $f_{(u, v)}(0)=0, f_{(u, v)}(+\infty)=-\infty, f_{(u, v)}^{\prime}(0)=0$, and $f_{(u, v)}^{\prime \prime}(0)>0$, there holds $f_{(u, v)}(t)>0$ for $t>0$ small and $f_{(u, v)}(t)<0$ for $t$ large. Therefore,

$$
\max _{t \geq 0} f_{(u, v)}(t)>0
$$

and it is achieved at some $t=\psi(u, v)>0$. Let

$$
\varphi(u, v):=\max _{t \geq 0} f_{(u, v)}(t) .
$$

Solving $f_{(u, v)}^{\prime}(\psi(u, v))=0$, we obtain

$$
\begin{aligned}
& \psi(u, v) \\
& =\left(\left(\int_{\mathbb{R}^{N}}\left(|\nabla u|^{2}+V(x) u^{2}+|\nabla v|^{2}+V(x) v^{2}\right)\right)\right. \\
& \quad \times\left(\int _ { \mathbb { R } ^ { N } } \left(\mu|u|^{p+2}+\mu|v|^{p+2}\right.\right. \\
& \left.\left.\left.+\sum_{k=1}^{m}\left(\alpha_{k}+\beta_{k}\right)|u|^{p_{k}}|v|^{q_{k}}\right)\right)^{-1}\right)^{1 / p} .
\end{aligned}
$$

Recalling (24), we can see $(\psi(u, v) u, \psi(u, v) v) \in N_{1}$.

To conclude, for each $(u, v) \in T_{1}$, there hold

$$
\begin{aligned}
I(\psi(u, v) u, \psi(u, v) v)= & \max _{t \geq 0} I(t u, t v)=\varphi(u, v)>0, \\
& (\psi(u, v) u, \psi(u, v) v) \in N_{1} .
\end{aligned}
$$

Define

$$
\begin{gathered}
d:=\inf _{(u, v) \in T_{1}} \varphi(u, v)=\inf _{(u, v) \in T_{1}} \max _{t \geq 0} I(t u, t v), \\
c:=\inf _{(u, v) \in N_{1}} I(u, v) .
\end{gathered}
$$


Proposition 7. Consider $d=c \geq 0$.

Proof. Firstly, for all $(u, v) \in N_{1}$,

$I(u, v)$

$$
=\frac{p}{2(p+2)} \int_{\mathbb{R}^{N}}\left(|\nabla u|^{2}+V(x) u^{2}+|\nabla v|^{2}+V(x) v^{2}\right)>0,
$$

which implies $c \geq 0$.

Next, we show that $d=c$. On one hand, for all $(u, v) \in$ $N_{1} \subset T_{1}$, we know that $\psi(u, v)=1$ and $f_{(u, v)}^{\prime}(1)=0$, which imply

$$
I(u, v)=\max _{t \geq 0} I(t u, t v)=\varphi(u, v) \geq \inf _{(u, v) \in T_{1}} \varphi(u, v)=d .
$$

That is, $c \geq d$. On the other hand, for all $(u, v) \in T_{1}$,

$$
\max _{t \geq 0} I(t u, t v)=I(\psi(u, v) u, \psi(u, v) v) \geq c,
$$

where we have used the fact that $(\psi(u, v) u, \psi(u, v) v) \in N_{1}$. That is, $d \geq c$. Therefore, $d=c \geq 0$.

Lemma 8. $\varphi(u, v)$ is sequentially weakly lower semicontinuous on $N_{1}$ with respect to $E$.

Proof. Let $\left(u_{n}, v_{n}\right) \rightarrow\left(u_{0}, v_{0}\right)$ weakly in $E$. By the uniform boundedness theorem, $\left\{\left(u_{n}, v_{n}\right)\right\}$ is bounded in $E$. Moreover, since the embedding $E \hookrightarrow L^{p+2}\left(\mathbb{R}^{N}\right) \times L^{p+2}\left(\mathbb{R}^{N}\right)$ is compact (see $[2,3])$, there is a subsequence $\left(u_{n}, v_{n}\right) \rightarrow\left(u_{0}, v_{0}\right)$ strongly in $L^{p+2}\left(\mathbb{R}^{N}\right) \times L^{p+2}\left(\mathbb{R}^{N}\right)$. By using Fatou's lemma, we obtain

$$
\begin{aligned}
\varphi\left(u_{0}, v_{0}\right) & =\max _{t \geq 0} I\left(t u_{0}, t v_{0}\right) \\
& =I\left(\psi\left(u_{0}, v_{0}\right) u_{0}, \psi\left(u_{0}, v_{0}\right) v_{0}\right) \\
& \leq \liminf _{n \rightarrow \infty} I\left(\psi\left(u_{0}, v_{0}\right) u_{n}, \psi\left(u_{0}, v_{0}\right) v_{n}\right) \\
& \leq \liminf _{n \rightarrow \infty} \max _{t \geq 0} I\left(t u_{n}, t v_{n}\right) \\
& =\liminf _{n \rightarrow \infty} \varphi\left(u_{n}, v_{n}\right)
\end{aligned}
$$

which ends the proof.

Lemma 9. $c$ is achieved on $N_{1}$.

Proof. Let $\left\{\left(u_{n}, v_{n}\right)\right\} \subset N_{1}$ be a minimizing sequence for $c$; that is, $\lim _{n \rightarrow \infty} I\left(u_{n}, v_{n}\right)=c$. We have $I\left(u_{n}, v_{n}\right)=(p / 2(p+$ 1)) $\int_{\mathbb{R}^{N}}\left(\left|\nabla u_{n}\right|^{2}+V(x) u_{n}^{2}+\left|\nabla v_{n}\right|^{2}+V(x) v_{n}^{2}\right)=(p / 2(p+$ 1)) $\left(\left\|u_{n}\right\|_{E}^{2}+\left\|v_{n}\right\|_{E}^{2}\right)$, which implies $\left\{\left(u_{n}, v_{n}\right)\right\}$ is bounded in E. Since $E \hookrightarrow L^{p+2}\left(\mathbb{R}^{N}\right) \times L^{p+2}\left(\mathbb{R}^{N}\right)$ compactly, up to a subsequence, there is $\left(u_{0}, v_{0}\right) \in E$ satisfying

$$
\begin{aligned}
& \left(u_{n}, v_{n}\right) \rightarrow\left(u_{0}, v_{0}\right) \quad \text { weakly in } E, \\
& \left(u_{n}, v_{n}\right) \longrightarrow\left(u_{0}, v_{0}\right),
\end{aligned}
$$

strongly in $L^{p+2}\left(\mathbb{R}^{N}\right) \times L^{p+2}\left(\mathbb{R}^{N}\right)$.
By using Lemma $6,\left\|u_{n}\right\|_{p+2}^{p+2}+\left\|v_{n}\right\|_{p+2}^{p+2} \geq \epsilon_{2}$, we have $\left(u_{0}, v_{0}\right) \neq$ $(0,0)$. Hence, $\left(u_{0}, v_{0}\right) \in T_{1}$.

Using Lemma 8, Proposition 7, and the fact that $\varphi\left(u_{n}, v_{n}\right)=I\left(u_{n}, v_{n}\right)$, we obtain

$$
\begin{gathered}
c=d \leq \varphi\left(u_{0}, v_{0}\right) \leq \liminf _{n \rightarrow \infty} \varphi\left(u_{n}, v_{n}\right)=\liminf _{n \rightarrow \infty} I\left(u_{n}, v_{n}\right)=c, \\
\varphi\left(u_{0}, v_{0}\right)=c .
\end{gathered}
$$

By using (36)

$$
c \leq \varphi\left(\psi\left(u_{0}, v_{0}\right) u_{0}, \psi\left(u_{0}, v_{0}\right) v_{0}\right)=\varphi\left(u_{0}, v_{0}\right)=c .
$$

Let

$$
\left(u_{c}, v_{c}\right):=\left(\psi\left(u_{0}, v_{0}\right) u_{0}, \psi\left(u_{0}, v_{0}\right) v_{0}\right) \text {. }
$$

We obtain $\left(u_{c}, v_{c}\right) \in N_{1}$ and it is a minimizer for $c$.

Lemma 10. $\left(u_{c}, v_{c}\right)$ defined as in (44) is a critical point of I. Proof. Define

$$
\begin{aligned}
& G(u, v) \\
& :=\int_{\mathbb{R}^{N}}\left(|\nabla u|^{2}+V(x) u^{2}+|\nabla v|^{2}+V(x) v^{2}\right) d x \\
& \quad-\int_{\mathbb{R}^{N}}\left(\mu|u|^{p+2}+\mu|v|^{p+2}\right. \\
& \left.\quad+\sum_{k}\left(\alpha_{k}+\beta_{k}\right)|u|^{p_{k}}|v|^{q_{k}}\right) d x .
\end{aligned}
$$

From the proof of Lemma 9, we know that $\left(u_{c}, v_{c}\right)$ is a minimizer for $\min _{(u, v) \in T_{1}} I(u, v)$ under the constrained condition $G(u, v)=0$. Hence, there is a Lagrange multiplier $\lambda$ such that

$$
\left(d I\left(u_{c}, v_{c}\right)+\lambda d G\left(u_{c}, v_{c}\right)\right)\left[\varphi_{1}, \varphi_{2}\right]=0, \quad \forall\left(\varphi_{1}, \varphi_{2}\right) \in E .
$$

Choosing $\left(\varphi_{1}, \varphi_{2}\right)=\left(u_{c}, 0\right)$, we have

$$
\begin{aligned}
& 0=\int_{\mathbb{R}^{N}}\left(\left|\nabla u_{c}\right|^{2}+V(x) u_{c}^{2}-\mu\left|u_{c}\right|^{p+2}\right. \\
& \left.\quad-\sum_{k=1}^{m} \alpha_{k}\left|u_{c}\right|^{p_{k}}\left|v_{c}\right|^{q_{k}}\right) d x \\
& +\lambda \int_{\mathbb{R}^{N}}\left(2\left|\nabla u_{c}\right|^{2}+2 V(x) u_{c}^{2}-(p+2) \mu\left|u_{c}\right|^{p+2}\right. \\
& \left.\quad-\sum_{k=1}^{m}\left(\alpha_{k}+\beta_{k}\right) p_{k}\left|u_{c}\right|^{p_{k}}\left|v_{c}\right|^{q_{k}}\right) d x .
\end{aligned}
$$


Choosing $\left(\varphi_{1}, \varphi_{2}\right)=\left(0, v_{c}\right)$, we have

$$
\begin{aligned}
& 0=\int_{\mathbb{R}^{N}}\left(\left|\nabla v_{c}\right|^{2}+V(x) v_{c}^{2}-\mu\left|v_{c}\right|^{p+2}\right. \\
&\left.-\sum_{k=1}^{m}\left(\alpha_{k}+\beta_{k}\right)\left|u_{c}\right|^{p_{k}}\left|v_{c}\right|^{q_{k}}\right) d x \\
&+\lambda \int_{\mathbb{R}^{N}}\left(2\left|\nabla v_{c}\right|^{2}+2 V(x) v_{c}^{2}-(p+2) \mu\left|v_{c}\right|^{p+2}\right. \\
&\left.\quad-\sum_{k=1}^{m}\left(\alpha_{k}+\beta_{k}\right) q_{k}\left|u_{c}\right|^{p_{k}}\left|v_{c}\right|^{q_{k}}\right) d x .
\end{aligned}
$$

Combining (47) with (48) and using $p_{k}+q_{k}=p+2$, we obtain

$$
\begin{aligned}
& 0=G\left(u_{c}, v_{c}\right)+2 \lambda G\left(u_{c}, v_{c}\right) \\
& -\lambda p \int_{\mathbb{R}^{N}}\left(\mu\left|u_{c}\right|^{p+2}+\mu\left|v_{c}\right|^{p+2}\right. \\
& \left.\quad+\sum_{k=1}^{m}\left(\alpha_{k}+\beta_{k}\right)\left|u_{c}\right|^{p_{k}}\left|v_{c}\right|^{q_{k}}\right) d x .
\end{aligned}
$$

By the fact that $\left(u_{c}, v_{c}\right) \in N_{1}$, namely, $G\left(u_{c}, v_{c}\right)=0$, that is, $\int_{\mathbb{R}^{N}}\left(\mu\left|u_{c}\right|^{p+2}+\mu\left|v_{c}\right|^{p+2}+\sum_{k=1}^{m}\left(\alpha_{k}+\beta_{k}\right)\left|u_{c}\right|^{p_{k}}\left|v_{c}\right|^{q_{k}}\right)=\left\|u_{c}\right\|_{E}^{2}+$ $\left\|v_{c}\right\|_{E}^{2}$, we obtain

$$
0=\lambda p\left(\left\|u_{c}\right\|_{E}^{2}+\left\|v_{c}\right\|_{E}^{2}\right) .
$$

Since $\left(u_{c}, v_{c}\right) \in T_{1},\left(u_{c}, v_{c}\right) \neq(0,0)$, which leads to $\lambda=0$. Therefore, $d I\left(u_{c}, v_{c}\right)=0$.

Let us now give a proof of Theorem 1 .

Proof of Theorem 1. Lemma 10 shows that $\left(u_{c}, v_{c}\right)$ is the critical point of energy functional $I$. By Lemma $5,\left(u_{c}, v_{c}\right)$ is a solution of system (10). We now claim that $u_{c} \not \equiv \quad 0$ and $v_{c} \not \equiv 0$. Hence $\left(\left|u_{c}\right|,\left|v_{c}\right|\right)$ is a ground state of system (10).

Assume for contradiction that $u_{c} \not \equiv 0, v_{c} \equiv 0$. On one hand, one can see that $u_{c}$ is a solution of scalar equation

$$
-\Delta u+V(x) u=\mu|u|^{p} u .
$$

Using Lemma 9 and Proposition 7, we have $d=c=I\left(u_{c}, 0\right)=$ $J\left(u_{c}\right)$, where

$$
J(u):=\frac{1}{2} \int_{\mathbb{R}^{N}}\left(|\nabla u|^{2}+V(x) u^{2}\right) d x-\frac{\mu}{p+2} \int_{\mathbb{R}^{N}}|u|^{p+2} d x .
$$

Let $M=\left\{v \in H^{1}\left(\mathbb{R}^{N}\right) \mid \int_{\mathbb{R}^{N}} V(x) v^{2} d x<+\infty, \int_{\mathbb{R}^{N}}\left(|\nabla v|^{2}+\right.\right.$ $\left.\left.V(x) v^{2}\right) d x=\mu \int_{\mathbb{R}^{N}} v^{p+2} d x\right\}$. Let $w$ be the ground state of (51) (see [14] for the existence). There hold

$$
\int_{\mathbb{R}^{N}}\left(|\nabla w|^{2}+V(x) w^{2}\right) d x=\mu \int_{\mathbb{R}^{N}} w^{p+2} d x
$$

and the least energy

$$
\begin{aligned}
J_{0} & :=\min _{v \in M} J(v)=J(w) \\
& =\frac{p}{2(p+2)} \int_{\mathbb{R}^{N}}\left(|\nabla w|^{2}+V(x) w^{2}\right) d x>0 .
\end{aligned}
$$

By the fact that $u_{c} \in M$ and (54), we know that

$$
J_{0} \leq J\left(u_{c}\right)=c
$$

Notice that $(\psi(w, w) w, \psi(w, w) w) \in N_{1}$. Using (24), (53), (13), and (14), we get $2 \mu+\sum_{k=1}^{m}\left(\alpha_{k}+\beta_{k}\right)>0$ and

$$
\psi(w, w)=\left(\frac{2 \mu}{2 \mu+\sum_{k=1}^{m}\left(\alpha_{k}+\beta_{k}\right)}\right)^{1 / p}<\frac{1}{2}<1 .
$$

By using the definition of $c$, (53), (54), and (56), we compute

$$
\begin{aligned}
c \leq & I(\psi(w, w) w, \psi(w, w) w) \\
= & \frac{\psi(w, w)^{2}}{2} \int_{\mathbb{R}^{N}} 2\left(|\nabla w|^{2}+V(x) w^{2}\right) d x \\
& -\frac{\psi(w, w)^{p+2}}{p+2} \int_{\mathbb{R}^{N}}\left(2 \mu|w|^{p+2}+\sum_{k}\left(\alpha_{k}+\beta_{k}\right)|w|^{p+2}\right) d x \\
= & \psi(w, w)^{2} J_{0} \\
& \times\left(\frac{2(p+2)}{p}-\frac{\psi(w, w)^{p} \cdot 2\left(2 \mu+\sum_{k}\left(\alpha_{k}+\beta_{k}\right)\right)}{\mu p}\right) \\
= & 2 \psi(w, w)^{2} J_{0} \\
< & J_{0},
\end{aligned}
$$

which contradicts (55).

Hence, we obtain $u_{c} \not \equiv 0$ and $v_{c} \not \equiv 0$. Therefore $\left(\left|u_{c}\right|,\left|v_{c}\right|\right)$ is a ground state of (10).

\section{Symmetry of Ground State}

In this section we first use a bootstrap argument similar to in [20] (see also Theorem 8.1.1 of [21]) to obtain a regularity result (Theorem 3 ). Then we use this regularity result to prove that the ground state of (16) is symmetric about origin.

Lemma 11. Let $u, v \in L^{q}\left(\mathbb{R}^{3}\right)$ (for some $p+1<q<\infty$ ) be a solution of (16). Then $u, v \in W^{2, q /(p+1)}\left(\mathbb{R}^{3}\right)$.

Proof. Since $u, v \in L^{q}\left(\mathbb{R}^{3}\right)$, we have

$$
|u|^{p} u,|v|^{p} v,|u|^{p_{k}-2}|v|^{q_{k}} u,|u|^{p_{k}}|v|^{q_{k}-2} v \in L^{q /(p+1)}\left(\mathbb{R}^{3}\right),
$$

by using Hölder's inequality. By the fact that $\left(-\Delta+|x|^{2}\right)$ is a maximal accretive operator in $L^{q /(p+1)}$ (see Theorem 2.5 in [22]), we have

$$
\begin{aligned}
(-\Delta & \left.+|x|^{2}+1\right)^{-1} L^{q /(p+1)}\left(\mathbb{R}^{3}\right) \\
& =\left\{\left.f \in W^{2, q /(p+1)}\left(\mathbb{R}^{3}\right)|| x\right|^{2} f \in L^{q /(p+1)}\left(\mathbb{R}^{3}\right)\right\},
\end{aligned}
$$

which implies $u, v \in W^{2, q /(p+1)}\left(\mathbb{R}^{3}\right)$. 
Proof of Theorem 3. Consider the sequence $q_{j}$ defined by

$$
\frac{1}{q_{j}}=(p+1)^{j}\left(\frac{1}{p+2}-\frac{2}{3 p}+\frac{2}{3 p(1+p)^{j}}\right) .
$$

Set $\delta=2 / 3 p-1 /(p+2)=(4-p) / 3 p(p+2)$. Since $p<4$, $\delta>0$. Direct computation shows

$$
\frac{1}{q_{j+1}}-\frac{1}{q_{j}}=-(p+1)^{j} p \delta \leq-\delta,
$$

that is to say, $1 / q_{j}$ is decreasing and $1 / q_{j} \rightarrow-\infty(j \rightarrow \infty)$. Since $q_{0}=p+2>0$, it follows that there exists $j_{0} \geq 0$ such that

$$
\frac{1}{q_{l}}>0, \quad \forall 0 \leq l \leq j_{0} ; \frac{1}{q_{j_{0}+1}} \leq 0 .
$$

We claim that $u, v \in L^{q_{j_{0}}}\left(\mathbb{R}^{3}\right) . u, v \in H^{1}\left(\mathbb{R}^{3}\right)$, by Sobolev embedding $u, v \in L^{q_{0}}\left(\mathbb{R}^{3}\right)$. If $u, v \in L^{q_{l}}\left(\mathbb{R}^{3}\right)$, for some $l \leq$ $j_{0}-1$, we have by Lemma 11

$$
u, v \in W^{2, q_{l} /(p+1)}\left(\mathbb{R}^{3}\right) .
$$

Using Sobolev embedding again $u, v \in L^{q}\left(\mathbb{R}^{3}\right)$, for all $q \geq$ $q_{l} /(p+1)$ such that $1 / q \geq(p+1) / q_{l}-2 / 3=1 / q_{l+1}$. In particular, $u, v \in L^{q_{l+1}}\left(\mathbb{R}^{3}\right)$. By induction, $u, v \in L^{q_{j_{0}}}\left(\mathbb{R}^{3}\right)$.

Using Lemma 11 and Sobolev embedding again, we have

$$
\begin{array}{r}
u \in L^{q}\left(\mathbb{R}^{3}\right), \quad \forall q \geq \frac{q_{j_{0}}}{p+1} \\
\text { such that } \frac{1}{q} \geq \frac{p+1}{q_{j_{0}}}-\frac{2}{3}=\frac{1}{q_{j_{0}+1}}<0 .
\end{array}
$$

Therefore

$$
u, v \in \bigcap_{2 \leq q<\infty} L^{q}\left(\mathbb{R}^{3}\right) .
$$

Part (a) then follows form Lemma 11.

By Sobolev's embedding, $u, v \in W^{1, \infty}\left(\mathbb{R}^{3}\right)$, which implies $u, v$ are uniformly Lipschitz continuous. Since $u, v \in L^{2}\left(\mathbb{R}^{3}\right)$, there holds that $u, v$ decay to zero at infinity; that is, (b) is valid.

To prove (c), we first take derivative to obtain for every $j \in\{1,2,3\}$

$$
\begin{aligned}
& \left.-\Delta+|x|^{2}+1\right) u_{j} \\
= & -2 x_{j} u+\mu(p+1)|u|^{p} u_{j} \\
& +\sum_{k=1}^{m} \alpha_{k}\left(\left(p_{k}-1\right)|u|^{p_{k}-2}|v|^{q_{k}} u_{j}+q_{k}|u|^{p_{k}-2} u|v|^{q_{k}-1} v_{j}\right), \\
\left(-\Delta+|x|^{2}+1\right) v_{j} & -2 x_{j} v+\mu(p+1)|v|^{p} v_{j} \\
& +\sum_{k=1}^{m} \beta_{k}\left(\left(q_{k}-1\right)|v|^{q_{k}-2}|u|^{p_{k}} v_{j}+p_{k}|v|^{q_{k}-2} v|u|^{p_{k}-1} u_{j}\right) .
\end{aligned}
$$

By (a) and Sobolev's embedding, $u, v \in W^{1,4}\left(\mathbb{R}^{3}\right) \hookrightarrow$ $L^{\infty}\left(\mathbb{R}^{3}\right)$. Hence, $|u|^{p} u_{j},|u|^{p_{k}-2}|v|^{q_{k}} u_{j}$, and $|u|^{p_{k}-2} u|v|^{q_{k}-1} v_{j} \epsilon$ $L^{4}\left(\mathbb{R}^{3}\right)$. Using (a), we know that $u, v \in L^{4(p+1)}\left(\mathbb{R}^{3}\right)$. By the fact that

$$
\begin{aligned}
(-\Delta & \left.+|x|^{2}+1\right)^{-1} L^{4}\left(\mathbb{R}^{3}\right) \\
& =\left\{\left.f \in W^{2,4}\left(\mathbb{R}^{3}\right)|| x\right|^{2} f \in L^{4}\left(\mathbb{R}^{3}\right)\right\}
\end{aligned}
$$

(see Theorem 2.5 in [22]), we have

$$
\int\left|x_{j} u\right|^{4} d x \leq \int\left(|x|^{2}|u|\right)^{4} d x<+\infty ;
$$

that is, $x_{j} u \in L^{4}\left(\mathbb{R}^{3}\right)$. Therefore, the right hand side of the first equation in (66) belongs to $L^{4}\left(\mathbb{R}^{3}\right)$. Using Lemma $11, u \in$ $W^{3,4}\left(\mathbb{R}^{3}\right) \hookrightarrow C^{2}\left(\mathbb{R}^{3}\right)$. Similarly, $v \in C^{2}\left(\mathbb{R}^{3}\right)$.

Proof of Corollary 4. This result is a corollary of Theorem 1 and Corollary 3 in [23]. By using Theorem 1, there exists a ground state $(u, v)$ of $(16)$. From Theorem 3 , the ground state is of class $C^{2}$ and satisfies $\lim _{|x| \rightarrow \infty} u=0, \lim _{|x| \rightarrow \infty} v=0$. The maximum principle applied to each single equation in (16) suggests that $u>0, v>0$. Then, by using Theorem 1 in [24] (see also Corollary 3 in [23]), all positive and decay solutions of (16) are radially symmetric about the origin, which proves this corollary.

\section{Conflict of Interests}

The author declares that there is no conflict of interests regarding the publication of this paper.

\section{Acknowledgments}

This research is supported by the National Natural Science Foundation of China no. 11201025 and the Fundamental Research Funds for the Central Universities.

\section{References}

[1] L. P. Pitaevskii and S. Stringari, Bose-Einstein Condensation, Clarendon Press, Oxford, UK, 2003.

[2] T. Bartsch and Z. Q. Wang, "Existence and multiple results for some superlinear elliptic problems on $\mathbb{R}^{N}$," Communications in Partial Differential Equations, vol. 20, pp. 1725-1741, 1995.

[3] D. G. Costa, "On a class of elliptic systems in $\mathbb{R}^{N}$," Electronic Journal of Differential Equations, vol. 7, pp. 1-14, 1994.

[4] A. Ambrosetti and E. Colorado, "Standing waves of some coupled nonlinear Schröinger equations," Journal of the London Mathematical Society, vol. 75, no. 1, pp. 67-82, 2007.

[5] H. Berestycki and P. Lions, "Nonlinear scalar field equations. I. Existence of a ground state," Archive for Rational Mechanics and Analysis, vol. 82, no. 4, pp. 313-345, 1983.

[6] W. Y. Ding and W. M. Ni, "On the existence of positive entire solutions of a semilinear elliptic equation," Archive for Rational Mechanics and Analysis, vol. 91, no. 4, pp. 283-308, 1986.

[7] V. Georgiev and G. Venkov, "Symmetry and uniqueness of minimizers of Hartree type equations with external Coulomb potential," Journal of Differential Equations, vol. 251, no. 2, pp. 420-438, 2011. 
[8] B. Gidas, W. M. Ni, and L. Nirenberg, "Symmetry of positive solutions of nonlinear elliptic equations in $\mathrm{R}^{N}$," Journal of Mathematical Analysis and Applications A, vol. 7, pp. 369-402, 1981.

[9] N. Ikoma and K. Tanaka, "A local mountain pass type result for a system of nonlinear Schrödinger equations," Calculus of Variations and Partial Differential Equations, vol. 40, no. 3-4, pp. 449-480, 2011.

[10] T. C. Lin and J. C. Wei, "Ground state of N coupled nonliear Schrödinger equations in $\mathbb{R}^{n}, n \leq 3$," Communications in Mathematical Physics, vol. 255, pp. 629-653, 2005.

[11] T. C. Lin and J. C. Wei, "Spikes in two-component systems of nonlinear Schröinger equations with trapping potentials," Journal of Differential Equations, vol. 229, no. 2, pp. 538-569, 2006.

[12] L. Ma and L. Zhao, "Uniqueness of ground states of some coupled nonlinear Schrödinger systems and their application," Journal of Differential Equations, vol. 245, no. 9, pp. 2551-2565, 2008.

[13] L. A. Maia, E. Montefusco, and B. Pellacci, "Positive solutions for a weakly coupled nonlinear Schrödinger system," Journal of Differential Equations, vol. 229, no. 2, pp. 743-767, 2006.

[14] P. H. Rabinowitz, "On a class of nonlinear Schrödinger equations," Zeitschrift für Angewandte Mathematik und Physik, vol. 43, no. 2, pp. 270-291, 1992.

[15] G. M. Wei, "Existence and concentration of ground states of coupled nonlinear Schrödinger equations," Journal of Mathematical Analysis and Applications, vol. 332, no. 2, pp. 846-862, 2007.

[16] M. Lucia and Z. Tang, "Multi-bump bound states for a system of nonlinear Schrödinger equations," Journal of Differential Equations, vol. 252, no. 5, pp. 3630-3657, 2012.

[17] B. Sirakov, "Least energy solitary waves for a system of nonlinear schröinger equations in $\mathrm{R}^{n}$," Communications in Mathematical Physics, vol. 271, no. 1, pp. 199-221, 2007.

[18] X. Song, "Stability and instability of standing waves to a system of Schrödinger equations with combined power-type nonlinearities," Journal of Mathematical Analysis and Applications, vol. 366, no. 1, pp. 345-359, 2010.

[19] W. A. Strauss, "Existence of solitary waves in higher dimensions," Communications in Mathematical Physics, vol. 55, no. 2, pp. 149-162, 1977.

[20] R. Fukuizumi and T. Ozawa, "Exponential decay of solutions to nonlinear elliptic equations with potentials," Zeitschrift für angewandte Mathematik und Physik ZAMP, vol. 56, no. 6, pp. 1000-1011, 2005.

[21] T. Cazenave, An Introduction to Nonlinear Schrödinger Equations, 1993.

[22] N. Okazawa, "An $L^{p}$ theory for Schrödinger operators with nonnegative potentials," Journal of the Mathematical Society of Japan, vol. 36, no. 4, pp. 675-688, 1984.

[23] B. Y. Liu and L. Ma, "Symmetry and uniqueness result for the Schrödinger system with potential," preprint.

[24] J. Busca and B. Sirakov, "Symmetry results for semilinear elliptic systems in the whole space," Journal of Differential Equations, vol. 163, no. 1, pp. 41-56, 2000. 


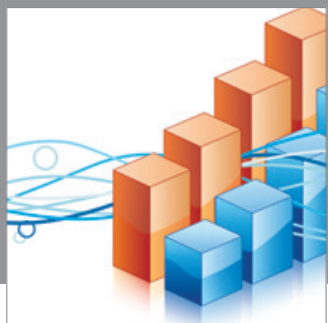

Advances in

Operations Research

mansans



The Scientific World Journal
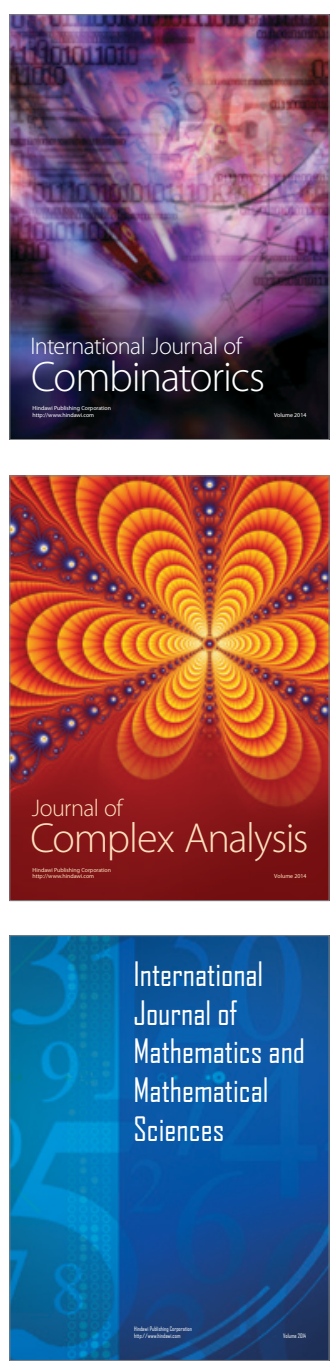
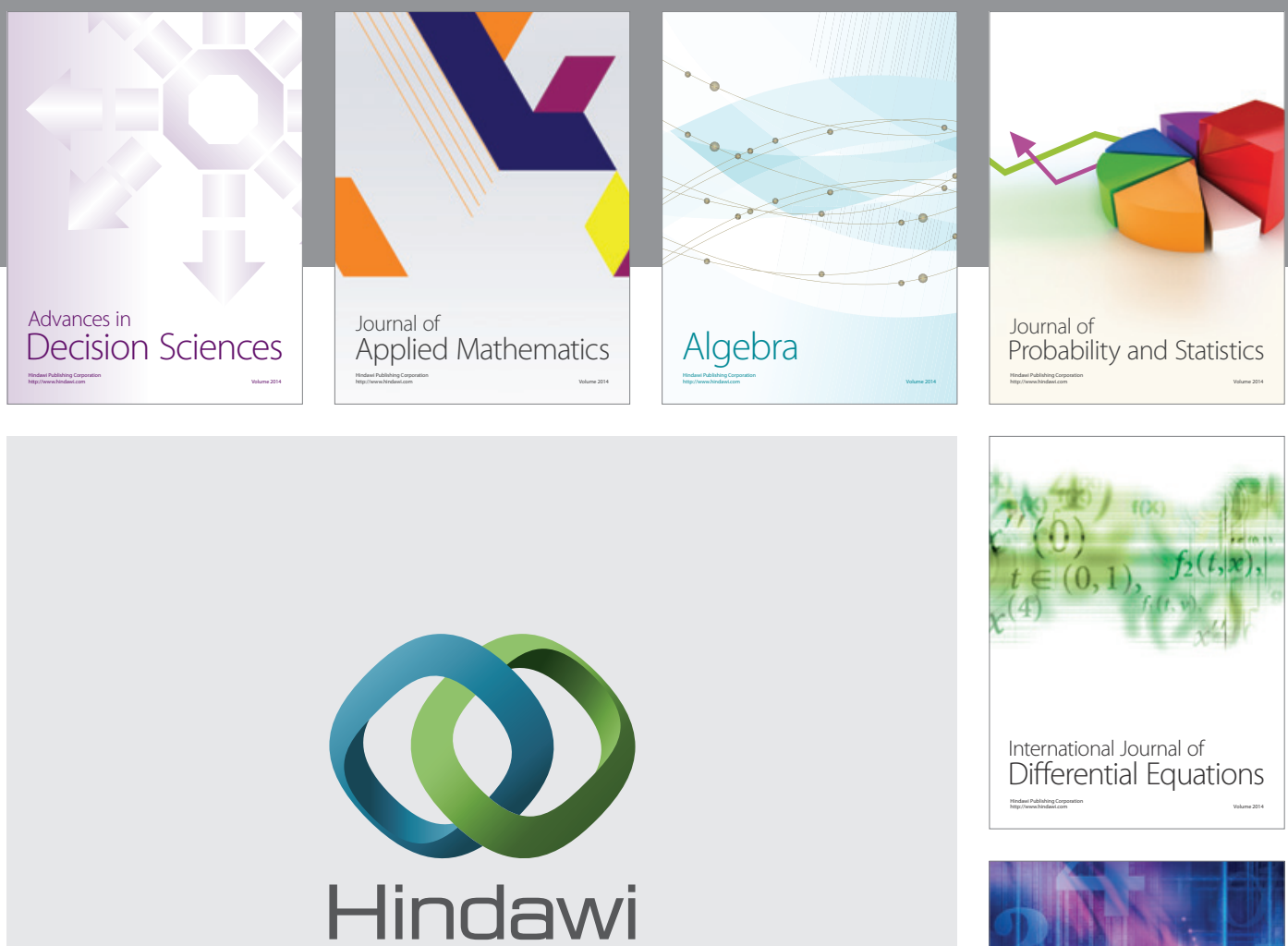

Submit your manuscripts at http://www.hindawi.com
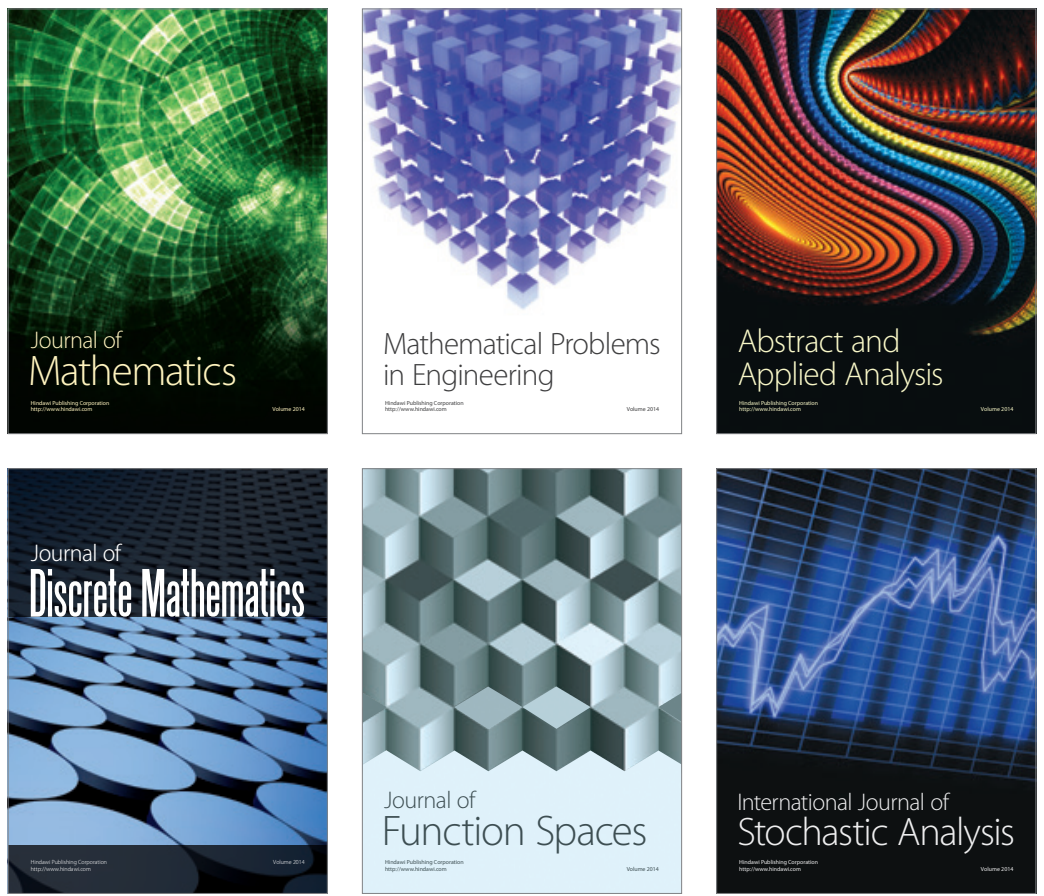

Journal of

Function Spaces

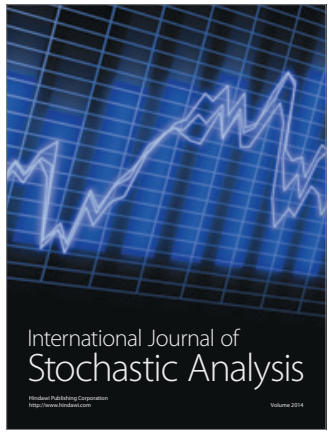

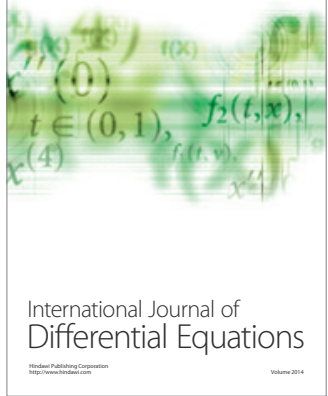
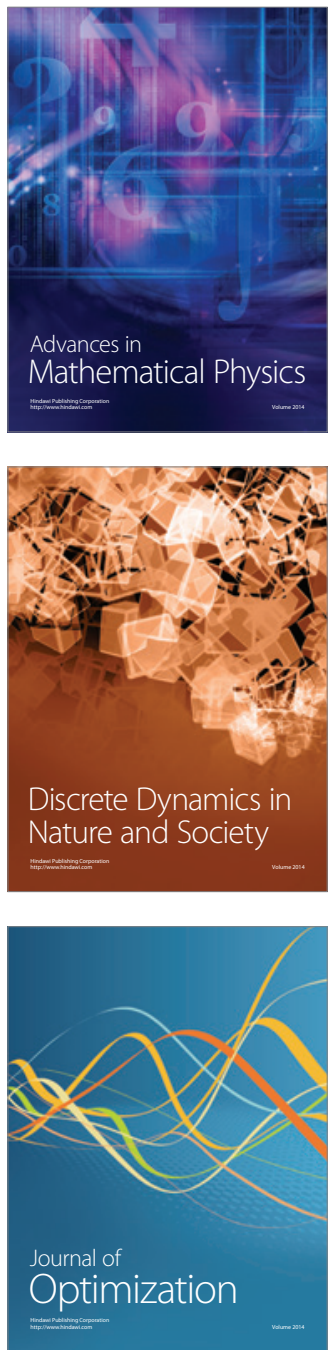